\title{
LA PREGUNTA COMO INSTRUMENTO DE LECTURA DE NARRACIONES
}

\author{
Javier González García \\ Universidad de Guanajuato (México)
}

\begin{abstract}
RESUMEN: En este artículo se analiza el uso de las demandas de información o preguntas, en un proceso de discusión que cuatro maestras llevan a cabo con sus alumnos a partir de la lectura de tres cuentos. La muestra está compuesta por dos aulas de dos escuelas públicas de Burgos capital (España), y dos más en Tampico (México). En cada clase hemos observado seis grupos de discusión compuesto por tres niños. Para ello, se plantea un estudio exploratorio a partir de una observación sistemática. En primer lugar, se elabora una descripción cualitativa de secuencias didácticas que muestran ejemplos del proceso de elaboración de preguntas, para terminar analizando y comparando de forma cuantitativa la evolución en el uso de las preguntas. El conocimiento de la capacidad de respuesta que tiene el niño, ayuda al profesor a decidir el tipo de apoyo que necesitará en la comprensión de la narración. Los resultados apuntan a que la variedad en los tipos de preguntas facilita la recuperación y elaboración del discurso en los niños. El presente estudio tiene un diseño exploratorio, puesto que se recurrió a grupos de comparación y no a grupos de control.
\end{abstract}

PALABRAS CLAVE: Preguntas, métodos educativos, tipos de información, narraciones, discusión.

\section{QUESTION AS A TOOL FOR READING STORIES}

\begin{abstract}
This article discusses the use of requests for information or questions, in a process of discussion that four teachers conducted with students from the reading of three stories. The sample consists of two classrooms in two public schools in the city of Burgos (Spain), and two more in Tampico (Mexico). In each class we have observed six focus groups composed of three children. To do this, we propose an exploratory study based on systematic observation. First, it produces a qualitative description of teaching sequences showing examples of process questions, to finish analyzing and comparing quantitatively the
\end{abstract}


evolution of the use of questions. Knowledge of responsiveness that has the child helps the teacher to decide what kind of support they need in understanding the narrative. The results suggest that the variety in the types of questions facilitates the recovery and processing of speech in children. This study is an exploratory design, was used as comparison and control groups.

KEYWORDS: Questions, teaching methods, information ways, story readings, discussion.

Recibido: 21/11/2011

Aceptado: 09/05/2012

\section{INTRODUCCIÓN}

Los maestros tienden a afirmar que el diálogo es un medio fundamental de enseñanza, pero son pocos los que lo ponen diariamente en práctica. Consideran que el tiempo que pueden dedicar a cada niño para conversar es limitado, y hay que emplearlo del mejor modo posible. La conversación con cada niño, o en grupos pequeños, no sólo permite apreciar las dificultades infantiles sino también las destrezas del propio maestro en el uso del lenguaje (Borzone, 2005). Escuchan al niño, y se proyectan sobre aquello que ofrezca posibilidades de ampliar el pensamiento infantil, eligiendo la forma más idónea de llevar a cada niño a interpretar de forma más amplia sus experiencias. Alrededor de los cinco y seis años muchos niños comienzan a adquirir conciencia de las necesidades que los demás aprecian como interlocutores, fruto de repetidas experiencias de conversación con adultos u otros niños mayores que ellos (Díez y Pardo, 2000).

Analizar los procesos de construcción conjunta del conocimiento en un diálogo puede ayudar a encontrar la forma de seleccionar estrategias, que ayuden al niño a pensar y a expresar su pensamiento mediante el uso del lenguaje. Se les puede ayudar a reconocer el tipo de respuesta que sugieren ciertas formas de pregunta, y prepararlos para que sepan distinguir alternativas. A la vez que se intenta estimularles a tomar iniciativa en el diálogo mediante la formulación de preguntas o explicándoles los puntos de desacuerdo.

Piaget (1977) sostenía que las preguntas ejercen una influencia esencial sobre el desarrollo cognitivo, y prefería sobre todo las preguntas que señalan contradicciones con lo que provocan al niño a pensar acerca de algo desde otro punto de vista. Las preguntas siempre han estado asociadas al modo cómo conocemos. De hecho, la filosofía se ha basado en aprender a hacer preguntas (Puig y Sátiro, 2000 y Signes, 2004); cada uno elabora su propio conocimiento, pero nadie lo puede hacer sin la ayuda de los demás. Cualquier pregunta puede actuar como guía del alumno, para que resuelva la tarea en un nivel superior al que alcanzaría sin ayuda (Tough, 1989).

Las demandas de información forman parte de un tipo característico de discurso, el educativo, en el que, curiosamente, quien conoce las respuestas es quien plantea las preguntas. También sirven como medio para acercarnos a la diversidad que existe en cada aula. La función de la curiosidad es impulsarnos a preguntar y a cuestio- 
nar el mundo en que vivimos, y al preguntar y preguntarnos podemos descubrir, crear y recrear diversas formas culturales.

Un profesor puede preguntar a sus alumnos sin necesidad de "interrogarlos", puede pensar preguntas para que sus alumnos sigan pensando y buscando respuestas (Fresquet, 2003). Existe un progresivo aprendizaje en interacción entre las maestras y los grupos de alumnos. Las maestras van aprendiendo a esperar respuestas y a formular sólo unas pocas preguntas (González, 2006). Aprender a esperar una respuesta es difícil, muchos maestros formulan excelentes preguntas, pero se "precipitan" respondiéndolas ellos mismos (González, 2007, 2008). Tal vez teman al silencio o al fracaso de los niños. Algunas maestras tienden a precipitarse debido, posiblemente, a que no esperan que los niños a esta edad sean capaces de justificar sus respuestas, piensan que "es pronto para que los niños puedan argumentar sus respuestas" (Díez, 2002).

Albanese y Antoniotti (1997) han estudiado la relación entre los componentes del estilo de diálogo y la comprensión de historias en niños. Estas autoras establecen medidas en torno a la habilidad para retener con precisión las historias, cuando varios componentes del diálogo son manipulados. Demuestran que el uso de cuestionarios durante la fase inicial de las narraciones aumenta su retención. Para ello hacen una clasificación en seis tipos de preguntas que están mediando en la comprensión de una lectura previa.

1. Enciclopédicas: revisan un concepto, idea o palabra nueva que influye en el desarrollo de la comprensión de un texto.

2. De verificación: constatan el nivel de comprensión de los interlocutores.

3. Personales: demandan situaciones reales análogas a la del texto, como anécdotas o episodios personales.

4. Inferenciales: son demandas que organizan una secuenciación lógica y temporal del texto previo.

5. De opinión: preguntas abiertas que solicitan valoración en torno a lo leído o hablado.

6. Retóricas: al principio o final de un tópico o contenido del debate. Tienen la función de romper el hielo frente a una instrucción que la va a seguir. Suelen iniciar o poner fin a la discusión.

En otro trabajo anterior Albanese y Antoniotti (1992) realizan un estudio de la interacción entre niños y adultos, durante una lectura compartida de un libro en etapa preescolar. Los resultados demostraron que el uso del estilo de diálogo permite un más alto nivel de comprensión que el estilo narrativo. Además el estudio daba luz a tres características, todas con relación al uso de preguntas del maestro que distinguieron el estilo de interacción del adulto.

Pero el estilo educativo predominante en la escuela occidental ha sido el que Rogoff y Toma (1997) denominan "Modelo de transmisión de la instrucción". En este modelo el profesor transmite la información, el alumno la recibe y el profesor comprueba si esta transmisión ha tenido lugar. Este formato es denominado I.R.E. (Inicio, Respuesta y Evaluación) y en él es habitual que el profesor inicie un tema con una pregunta de la cual ya conoce la respuesta, un estudiante responda y el profesor eva- 
lúe esta respuesta. En contraste con este modelo de enseñanza, Rogoff (1993) define lo que ella denomina "Perspectiva de transformación de la participación". Este modelo de instrucción se centra en el aprendizaje a través de la construcción de las ideas con los otros en tareas compartidas, y en la que los papeles de los aprendices son concebidos como una "participación multifacética" en el transcurso de la actividad conjunta.

En este trabajo nos planteamos analizar el uso de preguntas, en un proceso de elaboración conjunta de la información a partir de cuentos que cuatro maestras llevan a cabo con sus alumnos. Para ello se plantea un estudio exploratorio a partir de una observación sistemática.

\section{MÉTODO}

\section{Muestra}

La grabación de la observación se realiza a los mismos alumnos, durante el último curso de Educación Infantil, es decir, a la edad de cinco a seis años, en tres tomas durante el curso 2005-2006. La muestra está formada por dos clases pertenecientes a dos escuelas públicas de Burgos (España), denominados grupo A y B, y otras dos en Tamaulipas (México), grupos C y D. Cada clase o grupo, es dividida en 6 subgrupos de 3 niños, que es como se desarrolla la actividad observada: maestra y tres niños. La decisión sobre qué niños forman parte de cada grupo se delega en las maestras, todas han seguido un criterio de azar para elegir la conformación de cada grupo de tres niños. Los niños no han presentado reactividad frente a la cámara de vídeo, admitiendo la presencia de un observador ajeno a la clase. Únicamente han surgido ocasionalmente comentarios espontáneos, respondidos con plena naturalidad por parte de las maestras. No ha sido considerada la variable sexo de los niños, por interesarnos explorar el proceso de construcción del conocimiento en general, pero sí apuntamos a diferencias entre niños y niñas, variable a observar en próximos trabajos. El estatus socioeconómico de los colegios es similar, insertados en barrios de clase media-baja, dominando la procedencia de familias de padres jóvenes. Los cuatro centros educativos comparten la apuesta por la lectura a través de programas curriculares específicos en animación y comprensión lectora.

Las observaciones se realizan en formato audiovisual al inicio en los meses de diciembre, febrero y mayo. En total han sido grabadas 72 sesiones, con un total de 11.614 turnos conversacionales en torno a la lectura de tres cuentos.

Las maestras establecen la misma actividad para todos los grupos de tres niños, que consiste en: 1) Elección del texto con unos objetivos de aprendizaje, 2) Cada maestra divide la clase en grupos de tres niños, 3) Lee el texto elegido y acompaña y estructura libremente todo el proceso de discusión.

La duración de la lectura previa ha sido de 3 a 4 minutos por parte de las maestras, esta fase no ha sido recogida en el análisis, quedando abierta para otras investigaciones. A continuación se establece una discusión, que es la parte analizada en esta investigación. Se han eliminado los dibujos y viñetas, reduciendo la lectura al texto escrito, para favorecer que los niños reaccionen únicamente a las 
palabras, y que las maestras aumenten el uso de distintos recursos de voz: inflexiones, cambios de ritmo y onomatopeyas, normalmente sustituidas por las pausas para mostrar cada ilustración. Es el niño quien va a elaborar sus propias imágenes a partir del texto. La forma de llevar a cabo la discusión, y los objetivos, se delegan en las maestras, para que sean ellas las que libremente den sentido y significado a la actividad propuesta.

El material utilizado han sido tres cuentos. Los criterios de selección de los tres textos narrativos (García Sánchez y Pacheco, 1978), ha seguido los criterios explicitados por Van Dijk y Kintsch (1983: 134): "claridad de la superestructura, cantidad de proporciones, grado de redundancia, grado de accesibilidad temática, calidad de la fuente, cualidades de estilo, novedad, pertinencia ética, léxico, y extensión".

\section{Entrevistas a las maestras}

Para cotejar y complementar los datos observados realizamos una entrevista a cada maestra. Intentamos constatar el estilo docente y el contexto previo del que partían, así como los objetivos que cada maestra se ha marcado durante la investigación. Dividimos la entrevista en tres bloques (Tabla 1). En el primero analizamos los estilos docentes de las maestras y cómo se traduce en el uso que cada una da a la discusión y a la narración. En el segundo ofrecemos información acerca del guión de objetivos que cada maestra se ha marcado. La información recogida aporta aspectos de sus biografías profesionales y de sus teorías implícitas sobre el uso que dan y esperan de la lectura, del diálogo y del trabajo en grupo (Tabla 2).

Tabla 1. Guión de la entrevista

\section{Bloque biográfico: "La transmisión de estilos"}

1) ¿Cuántos años llevas trabajando la puesta en discusión en clase?

2) ¿Con qué edades?

3) ¿En este colegio?

4) ¿Utilizas el trabajo en grupo?, ¿en qué momentos?

5) ¿Qué sistema de trabajo has seguido?

6) ¿Qué uso haces de los libros de texto?

7) ¿Y de los cuentos?

8) ¿Qué es para ti el cuento?

9) ¿Para qué sirve?

10) ¿Cómo usarlo?

11) ¿Cómo lo has usado en esta ocasión?

Bloque de contraste: "objetivos que se han marcado"

12) ¿Cuál ha sido el objetivo prioritario de la tarea?

13) ¿Qué otros objetivos importantes percibes?

14) ¿Qué crees haber conseguido? 
Tabla 2. Entrevista "Los estilos docentes"

\begin{tabular}{|c|c|}
\hline MAESTRA A & MAESTRA B \\
\hline \multicolumn{2}{|c|}{ 1. Biografía } \\
\hline $\begin{array}{l}30 \text { años como maestra. } \\
20 \text { años como maestra de Educación Infantil. } \\
9 \text { años en el Centro A. }\end{array}$ & $\begin{array}{l}\text { Comienzos en Educación Compensatoria, } \\
\text { tuvo alumnos de } 3 \text { a } 14 \text { años. } \\
\text { Más tarde pasa a una escuela unitaria. } \\
5 \text { años como maestra de Educación Infantil } \\
\text { ( } 2 \text { como profesora de apoyo). } \\
3 \text { años en el Centro B. }\end{array}$ \\
\hline \multicolumn{2}{|c|}{ 2. Estilos docentes } \\
\hline $\begin{array}{l}10 \text { primeros años sigue método transmisivo. } \\
\text { Al integrarse en Educación Infantil, con una } \\
\text { población estable de alumnos, comienza a } \\
\text { aplicar el trabajo cooperativo y la discusión. } \\
\text { Tras ingresar en el centro A, se va formando } \\
\text { en metodologías constructivistas. }\end{array}$ & $\begin{array}{l}\text { Comienza por un sistema transmisivo. } \\
\text { Descubre la importancia del trabajo } \\
\text { cooperativo. } \\
\text { Al integrarse en Educación Infantil, descubre } \\
\text { un método de acompañamiento. }\end{array}$ \\
\hline \multicolumn{2}{|c|}{ 3. Trabajo en grupo } \\
\hline $\begin{array}{l}\text { La clave está en el tipo de agrupaciones, que } \\
\text { depende del tipo de actividad. } \\
\text { Agrupamientos que utiliza: } \\
\text { - Asamblea o gran grupo para rutinas y } \\
\text { actividades abiertas, como es la lectura de } \\
\text { cuentos. } \\
\text { - Rincones o talleres para trabajar temas } \\
\text { específicos. En grupos de tres y por } \\
\text { parejas. } \\
\text { La presencia de la maestra no es necesaria en } \\
\text { algunas actividades y rutinas. }\end{array}$ & $\begin{array}{l}\text { El trabajo en grupo sirve para potenciar } \\
\text { valores: solidaridad, responsabilidad, etc. } \\
\text { Agrupamientos que utiliza: } \\
\text { - Asamblea para la lectura de cuentos. } \\
\text { - Las tareas del aula, como las fichas, son } \\
\text { realizados individualmente, estando los } \\
\text { niños divididos en grupos de } 6 \text {. } \\
\text { - Apenas trabaja por parejas o en grupos } \\
\text { reducidos. } \\
\text { La presencia de la maestra es necesaria en } \\
\text { todas las actividades. }\end{array}$ \\
\hline \multicolumn{2}{|c|}{ 4. Uso de la discusión y de la argumentación } \\
\hline $\begin{array}{l}\text { En este tipo de actividades de debate puede } \\
\text { y debe darse la argumentación, especialmente } \\
\text { si te marcas como objetivo el aumento y } \\
\text { desarrollo del vocabulario. } \\
\text { Algunos niños ejercen de portavoces, } \\
\text { explicando palabras y conceptos al resto de } \\
\text { su grupo. } \\
\text { De la lectura repetida y la discusión, se } \\
\text { percibe un aprendizaje y unas expectativas. }\end{array}$ & $\begin{array}{l}\text { Los niños no son capaces de fundamentar lo } \\
\text { suficiente como pueda ser más adelante, con } \\
\text { una mayor madurez de pensamiento y } \\
\text { lenguaje. } \\
\text { Es positivo sentar las bases e iniciar el } \\
\text { aprendizaje del proceso de argumentación a } \\
\text { estas edades. "Empezar desde esta etapa a } \\
\text { ejercitar la escucha y el respeto de turnos, } \\
\text { quizá nos ahorraría la pobreza de los debates } \\
\text { en los medios de comunicación". } \\
\text { Prevalece escuchar para luego participar en la } \\
\text { conversación. } \\
\text { La demanda de evaluación de los enunciados } \\
\text { de otros obtiene escasa continuidad. Contestar } \\
\text { a "¿qué opinas de lo que ha dicho tu } \\
\text { compañero?", supone haber escuchado y }\end{array}$ \\
\hline
\end{tabular}




\begin{tabular}{|c|c|}
\hline & $\begin{array}{l}\text { entendido lo que otro ha dicho, además de } \\
\text { tener un juicio para poder contrastar. } \\
\text { La confrontación de opiniones espontánea es } \\
\text { muy difícil, había que provocarlo. } \\
\text { Existe un contagio entre los miembros del } \\
\text { grupo a la hora de perderse en hablar de } \\
\text { detalles personales ajenos al cuento. } \\
\text { Asume la posibilidad de haber tenido que } \\
\text { hacer preguntas más concretas, para facilitar } \\
\text { intervenciones más centradas en el cuento. }\end{array}$ \\
\hline MAESTRA C & MAESTRA D \\
\hline \multicolumn{2}{|c|}{ 1. Biografía } \\
\hline $\begin{array}{l}30 \text { años como maestra de Educación Infantil. } \\
3 \text { años en el Centro } C \text { del que es directora. }\end{array}$ & $\begin{array}{l}30 \text { años como maestra de Educación Infantil. } \\
5 \text { años en el Centro D. }\end{array}$ \\
\hline \multicolumn{2}{|c|}{ 2. Estilos docentes } \\
\hline $\begin{array}{l}10 \text { primeros años sigue método transmisivo. } \\
\text { Más tarde descubre método de } \\
\text { acompañamiento. } \\
\text { Para ir incorporando estrategias } \\
\text { constructivistas. Tras ingresar en el centro C, } \\
\text { va generando su concepto de "comunidades } \\
\text { de aprendizaje", donde integra a las familias } \\
\text { dentro del funcionamiento de su centro. }\end{array}$ & $\begin{array}{l}\text { Comienza por un sistema transmisivo, en el } \\
\text { que integra actividades de aprendizaje } \\
\text { significativo. } \\
\text { Gradualmente va incorporando estrategias } \\
\text { constructivistas. }\end{array}$ \\
\hline \multicolumn{2}{|c|}{ 3. Trabajo en grupo } \\
\hline $\begin{array}{l}\text { La clave está en el tipo de agrupaciones, que } \\
\text { depende del tipo de actividad. } \\
\text { Agrupamientos que utiliza: } \\
\text { - Grupos de cuatro niños para las rutinas } \\
\text { de clase. } \\
\text { - En asamblea para cerrar esas rutinas. }\end{array}$ & $\begin{array}{l}\text { El trabajo en grupo sirve para potenciar } \\
\text { valores: solidaridad, responsabilidad, etc. } \\
\text { Agrupamientos que utiliza: } \\
\text { - Las tareas del aula son realizadas } \\
\text { individualmente, distribuidos en grupos de } 6 \text {. } \\
\text { - Asamblea al finalizar cada tarea. }\end{array}$ \\
\hline \multicolumn{2}{|c|}{ 4. Uso de la discusión y de la argumentación } \\
\hline $\begin{array}{l}\text { En una discusión debe darse la argumentación, } \\
\text { especialmente si te marcas como objetivo la } \\
\text { comprensión y la escucha. } \\
\text { Da mucha importancia a la variable afectiva, } \\
\text { dando confianza y seguridad para que los } \\
\text { niños puedan verbalizar los problemas traen } \\
\text { de sus casas. (Muchos de sus alumnos } \\
\text { proceden de familias desestructuradas). }\end{array}$ & $\begin{array}{l}\text { Esta actividad desarrolla la capacidad de } \\
\text { justificar las respuestas. Es positivo empezar a } \\
\text { estas edades. } \\
\text { Es difícil Ilevarlos a que hagan intervenciones } \\
\text { largas, y sobre todo a que confronten lo que } \\
\text { ha dicho otro compañero. }\end{array}$ \\
\hline
\end{tabular}

En su práctica cotidiana, la maestra A organiza el trabajo del aula por rincones, estableciendo actividades por grupos de tres $y$, sobre todo por parejas; y las maestras B, C y D trabajan por grupos de 6 niños en las mismas actividades, usan el cuen- 
to y su lectura de manera análoga, primero lo leen y luego establecen una discusión en asamblea. En el grupo A, la maestra suele solicitar voluntarios para hacer un resumen de la lectura previa, advirtiendo que tienden a salir los mismos niños, en torno a ocho del total de 23 niños. De manera más puntual trabaja la creación a partir de actividades abiertas como: poner título, acabar un cuento ya empezado, o darlo continuidad en un punto determinado. En su aula hay un gran desarrollo de la expresión plástica y el juego. Uno de los rincones, la esquina más amplia del aula, es la biblioteca, con numerosos libros, y al lado un rincón dedicado a la lectoescritura con actividades como las "letras móviles", piezas de cartón o plástico con las que los niños van reproduciendo palabras y frases. Además ejercita una lectura silenciosa e individual en la que voluntariamente responden sobre el cuento que han elegido, sus preferencias, sus puntos de vista, para el resto de compañeros. En aplicación y seguimiento del sistema constructivista, existe el rincón de lectura por parejas, dentro de la organización del trabajo por rincones. En el grupo D como actividad extraescolar, se realiza periódicamente un taller de cuentos, donde se combinan marionetas y sombras, jornadas que se abren a los padres.

En los grupos B y C las maestras finalizan cada mañana con la lectura de un cuento, normalmente elegido y Ilevado al aula por uno de los alumnos. En ocasiones el cuento es Ilevado a explicación en asamblea, donde dominan las demandas de los niños a la maestra, que va dando los turnos. La maestra B hace hincapié en la dificultad de comunicarse individualmente con los niños o en grupos pequeños como es el caso de esta investigación. Esta maestra es responsable de un grupo de 26 niños, considera que si emplea todo su tiempo con cada niño o con cada tríada, los demás no tendrán posibilidad de tomar parte. Declara su necesidad de mantener la atención sobre el grupo entero, de ahí su preferencia de sacar la actividad fuera del aula. El sistema de trabajo por rincones que ejercita diariamente la maestra A parece adaptarse mejor a estos criterios. La maestra B percibe la posibilidad de este tipo de actividad en pequeño grupo, sólo durante la presencia de la profesora de apoyo, si no, no es partidaria de dejar a los niños solos.

Las cuatro maestras han sido conscientes del tipo de tarea que pueden llevar a cabo en un pequeño grupo de niños que participan juntos en una misma actividad, y la contribución de la conversación cuando ellas no están presentes. Los niños necesitan hablar espontáneamente, junto a una dirección y unos objetivos.

\section{Categorización}

Se escribe literalmente el diálogo mantenido entre los niños y su maestra, indicando entre paréntesis los componentes prosódicos: pausas, entonación; y paralingüísticos: gestos de afirmación o negación, señales con el dedo y brazos, movimientos de cabeza o corporales, que transmiten significados a través de canales no verbales. Una vez recogidas todas las grabaciones se transcribe el material audiovisual, y se elabora el sistema de categorías a partir de protocolos de observación, que tras numerosas modificaciones se adecua a los objetivos de esta investigación. Éstas nacen a partir de definir y operativizar nuestra unidad de análisis, turno conversacional, que forma parte de una unidad molar de observación: cada sesión de trabajo con cada cuento y grupo de tres niños. El proceso de categorización con- 
siste en un primer registro en forma de lista de rasgos que se sistematiza progresivamente. Intentamos que las categorías del sistema, además de cumplir con los requisitos de "exhaustividad" y de "exclusividad" (Anguera, 1992), sean flexibles y se hallen totalmente definidas.

1. Generamos inductivamente los criterios de observación más relevantes, a partir de la revisión del material audiovisual.

2. Agrupamos estos criterios para definir las diferentes dimensiones de análisis que vamos a utilizar.

3. Volvemos a las conversaciones con esas dimensiones de análisis para observar cómo varían internamente y discriminamos las categorías que las componen.

4. Compatibilizamos las dimensiones de análisis acotando sus criterios para que las categorías de una dimensión de análisis fueran mutuamente excluyentes, mientras que las categorías de diferentes dimensiones de análisis no lo fueran. Esto permite observar las múltiples combinaciones de categorías, es decir, su multifuncionalidad.

5. Tras la elaboración del sistema de categorías codificamos cada una de las sesiones grabadas. Se lleva a cabo por el investigador principal junto con dos psicólogos, quienes ajenos a la construcción de las categorías y a los fines de la investigación, presentan una posición objetiva. El acuerdo interobservadores fue en torno al 98\% de los enunciados analizados entre el investigador principal y los otros dos psicólogos.

El criterio de observación objeto de estudio en este artículo son la forma de uso de las demandas de información o preguntas. Nos fijamos en el criterio inicio-continuidad para poder observar si las preguntas han servido para dar inicio o continuación a cada tema o tópico, comparar entre los estilos de las maestras, y qué resultado tiene en los niños. Tipo de iniciación y grado de espontaneidad permite o fomenta la maestra, informando sobre de quién parte la iniciativa del enunciado. Veamos ejemplos.

Tabla 3. Inicio demanda abierta

$\begin{array}{lll}2172 & \text { MAESTRA } & \text { ¿Qué os ha parecido, eh? } \\ 2173 & \text { TODOS } & \text { (Sonríen) } \\ 2174 & \text { MAESTRA } & \text { ¿Os ha gustado? } \\ 2175 & \text { ANDREA } & \text { Sí } \\ 2176 & \text { IKER } & \text { Sí } \\ 2177 & \text { MAESTRA } & \text { ¿Quién son las protagonistas de este cuento? }\end{array}$

El interlocutor abre el discurso a través de una pregunta o demanda de información, dando origen a un nuevo tópico o una nueva actividad. Tiende a producir una continuidad en los siguientes turnos, se abre el discurso con una intención clara de continuidad. Suele ser una estrategia propia de la maestra (Turnos 2172, $2174 ; 155)$. 
Tabla 4. Inicio demanda centrada

155 MAESTRA Bueno pues ahora me vas a contar qué es lo que más os ha gustado. ¿Quién quiere empezar?

Se contribuye a la conversación a través de un andamiaje abierto. El interlocutor mantiene, conserva, prolonga, desarrolla, extiende, amplia enunciados ya emitidos, para dar cohesión, y extender el discurso a otro interlocutor, o al resto del grupo. En ocasiones, un niño imita a la maestra, adoptando su rol, y hace una pregunta. Entonces la maestra extiende esa pregunta al resto del grupo, para que no esperen que sea ella la que responda (Turno 1238), consiguiendo que se implique el resto del grupo (Turnos 1239, 1241)

Tabla 5. Continuación demanda. Niños

\begin{tabular}{|lll}
\hline 1231 & ALVAR & Pues dice mi madre que nunca digas a un señor que no, que no... \\
1232 & MAESTRA & Que no le conozcas \\
1233 & ALVAR & Que dice: "toma un caramelo", y que no vaya porque si no me lleva \\
1234 & MAESTRA & Claro, te puede llevar. ¿Eso te ha dicho tu mama? \\
1235 & SILVIA & ¿Y si es malo, qué? \\
1236 & ALVAR & Por eso \\
1237 & JUAN & ¿Y si tiene veneno? \\
1238 & MAESTRA & ¿Qué pasa si tiene veneno? \\
1239 & SILVIA & ¿Si tiene veneno el caramelo? \\
1240 & MAESTRA & iAh!, ¿qué pasa si tiene veneno el caramelo? \\
1241 & ALVAR & Pues que se duerme, y se lleva un veneno que se muerde y le llevan \\
& & a enterrar
\end{tabular}

Las maestras generan el discurso a partir de preguntas, mientras proporcionan simultáneamente pistas eficaces para la información necesaria. Se introduce a los niños en un discurso compartido con el maestro. Como tal, encaja en el tipo de proceso educativo definido por "ZDP", Zona de Desarrollo Próximo, que se define como la distancia entre el nivel de desarrollo efectivo del alumno (aquello que es capaz de hacer por sí solo) y el nivel de desarrollo potencial (aquello que sería capaz de hacer con la ayuda de un adulto o un compañero más capaz), y así el conocimiento de los alumnos es guiado por las preguntas, las pistas e instancias de las maestras para conseguir profundizaciones de las que los alumnos por sí solos parecían incapaces. Es un mecanismo que exige que participen activamente en la creación de un conocimiento compartido, en lugar de limitarse a estar sentados y escuchar cómo habla la maestra (Turnos 4423-27). 
Tabla 6. Continuación demanda. Construcción conjunta

\begin{tabular}{|lll|}
\hline 4423 & ALICIA & ¿Y si cogieras un trozo de cielo, como es invisible? \\
4424 & MAESTRA & ¿Un trozo de cielo? \\
4425 & ALICIA & ¿No puedes? \\
4426 & MAESTRA & ¿Y para qué ibas a coger un trozo de cielo? \\
4427 & ALICIA & Para ser invisible \\
4428 & MAESTRA & ¿Ah!, para ser invisible \\
4429 & ALICIA & Además el espíritu es invisible \\
4430 & MAESTRA & ¿El espíritu? \\
4431 & ALICIA & Porque es un fantasma \\
\hline
\end{tabular}

Como vemos la maestra proporciona un tipo de andamiaje abierto porque va resituando la demanda inicial en función de las continuaciones de los niños (Tabla 6).

\section{Tipo de análisis}

Tras la elaboración del sistema de categorización, y el contraste de los datos a través de las entrevistas, analizamos y comparamos de forma cuantitativa la evolución de las fuentes de información, desplegadas por los cuatro grupos observados en la reconstrucción conjunta del cuento. Para ello se empleó el programa estadístico SPSS.

El presente estudio no tiene un diseño experimental, puesto que se recurrió a grupos de comparación y no a grupos de control, ya desde sus objetivos resulta meramente exploratorio y requiere ser replicado con la inclusión de estos grupos, con un seguimiento estricto de la intervención y de los avances de los niños a través de evaluaciones más frecuentes.

Comenzamos la exposición de resultados de la observación sistemática, partiendo de los porcentajes globales en cada grupo. Exponemos como primer acercamiento las distribuciones generales de los porcentajes de los turnos, tanto en la comparación entre los grupos A y B (España), como en los grupos C y D (México). 


\section{Resultados}

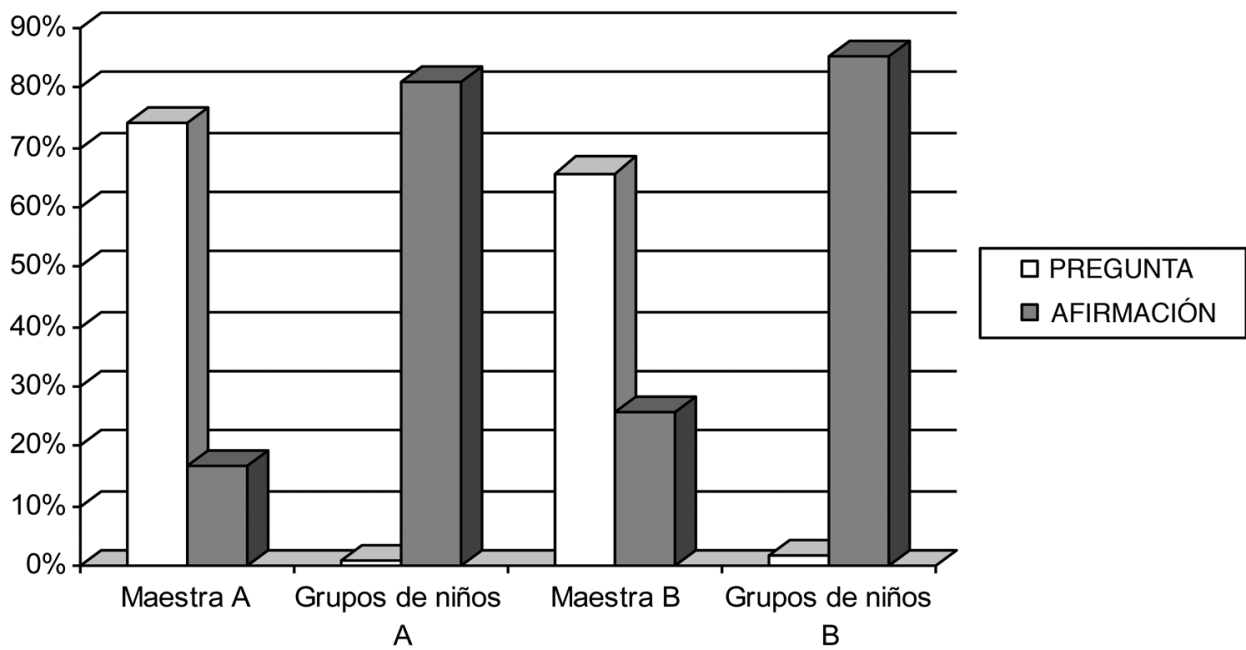

Figura 1. Porcentajes de preguntas y afirmaciones. Grupos $A$ y $B$

Las maestras demandan y elaboran información a partir de los enunciados de los niños, acción básica del andamiaje. En el grupo A es donde mejor ha podido observarse, ha sido clave saber dar continuidad a las respuestas iniciales del niño, dando voto de confianza que canaliza su necesidad de contar. De hecho, al sentirse escuchado, se centra mejor en las siguientes tareas. Es entonces cuando la maestra ha sacado a la luz ideas que estaban ya en la cabeza de cada niño y que sin su ayuda no hubieran sido expresadas.

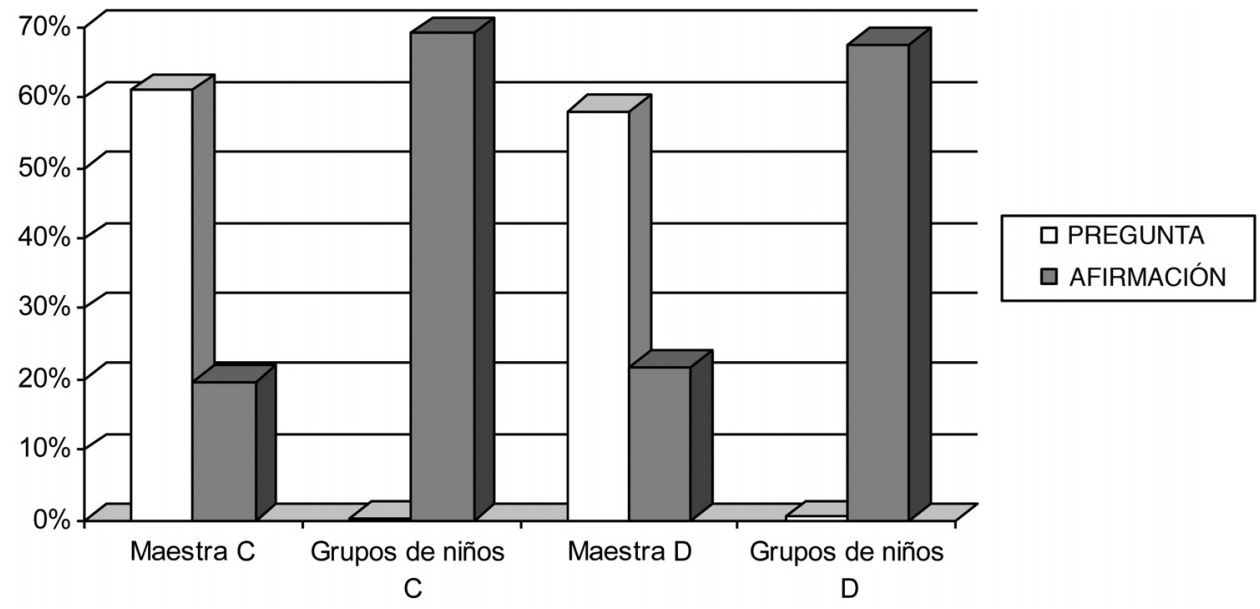

Figura 2. Porcentajes de preguntas y afirmaciones. Grupos $C$ y $D$ 
Las preguntas han sido claves en el proceso de participación. Hay que destacar el escaso porcentaje de preguntas o demandas de información en los niños de todos los grupos, que se debe a la dificultad que encierra para ellos, la identificación de la información adicional que necesitan.

Tabla 7. Evolución trimestral de las preguntas. Grupos $A$ y $B$

\begin{tabular}{|l|c|c|c|c|c|c|c|c|}
\hline \multirow{2}{*}{$\begin{array}{l}\text { CONTINUACIÓN } \\
\text { PREGUNTA }\end{array}$} & \multicolumn{2}{|c|}{ Cuento 1 } & \multicolumn{2}{c|}{ Cuento 2 } & \multicolumn{2}{c|}{ Cuento 3 } & \multicolumn{2}{c|}{ Total } \\
\cline { 2 - 9 } & Grupo A & Grupo B & Grupo A & Grupo B & Grupo A & Grupo B & Grupo A & Grupo B \\
\hline MAESTRA & $77,3 \%$ & $63,5 \%$ & $73,6 \%$ & $67 \%$ & $72,3 \%$ & $67,1 \%$ & $74,1 \%$ & $65,8 \%$ \\
\hline ALUMNO & $0,3 \%$ & $1,1 \%$ & $0,5 \%$ & $1,9 \%$ & $1,4 \%$ & $1,6 \%$ & $0,8 \%$ & $1,5 \%$ \\
\hline N turnos centrados & 682 & 1247 & 782 & 1153 & 1061 & 1042 & 2525 & 3442 \\
\hline
\end{tabular}

Normalmente es la maestra quien se sirve de esta estrategia de participación, $74,1 \%$ del total de intervenciones con leve descenso en A, 65,8\% con un ligero aumento en B. En los niños apenas se aprecia, en el grupo A parte de la práctica inexistencia del primer trimestre, y va aumentando hasta casi igualar al grupo B $(1,6 \%)$, en el último trimestre. Son unos porcentajes muy estables que siguen apuntando a una estructura de participación semidirectiva promovida por ambas maestras. Resultados muy parecidos en los grupos C y D, si bien, hay un menor porcentaje de preguntas en los niños.

Tabla 8. Evolución trimestral de las preguntas. Grupos $C$ y $D$

\begin{tabular}{|l|c|c|c|c|c|c|c|c|}
\hline \multirow{2}{*}{$\begin{array}{l}\text { CONTINUACIÓN } \\
\text { PREGUNTA }\end{array}$} & \multicolumn{2}{|c|}{ Cuento 1 } & \multicolumn{2}{c|}{ Cuento 2 } & \multicolumn{2}{c|}{ Cuento 3 } & \multicolumn{2}{c|}{ Total } \\
\cline { 2 - 9 } & Grupo C & Grupo D & Grupo C & Grupo D & Grupo C & Grupo D & Grupo C & Grupo D \\
\hline MAESTRA & $61,3 \%$ & $71,5 \%$ & $63,1 \%$ & $70,1 \%$ & $62,3 \%$ & $72,1 \%$ & $62,1 \%$ & $71,8 \%$ \\
\hline ALUMNO & $0,2 \%$ & $0,6 \%$ & $0,3 \%$ & $0,9 \%$ & $0,3 \%$ & $1,1 \%$ & $0,3 \%$ & $0,9 \%$ \\
\hline N turnos centrados & 542 & 447 & 767 & 653 & 1061 & 1042 & 2250 & 2342 \\
\hline
\end{tabular}

\section{CONCLUSIONES}

Las demandas de información son "índices de la actividad discursiva" (Pontecorvo y Orsolini, 1992) que sirven para construir la continuidad necesaria en una discusión. La maestra, con gran diferencia sobre el resto de participantes, es quien más demanda. Que un niño a esta edad demande información a otro es más difícil. Progresivamente lo van haciendo, siendo más capaces de "integrar la actividad propia" con la de otros compañeros (Díez, 2002). La forma de participación dominante es: la maestra pregunta-los alumnos responden. La clave está en encontrar un término medio a la hora de abrir las preguntas. Observamos cómo unas preguntas dan respuestas con mayor riqueza que otras: 
- Los niños pueden irse a lo primero que les pasa por la cabeza, contenidos ajenos a la lectura previa, cuando las demandas se abren mucho, como la pregunta: “ialgo más del cuento?".

- La pregunta abierta "¿Qué es lo que más te ha gustado del cuento?", que hemos revisado como una instrucción inicial de la actividad, suele ir acompañada por una respuesta de detalle que no contribuye a un debate de turnos fluidos.

- En cambio una pregunta con un mayor número de matizaciones, como "Imagínate que eres María, la niña invisible, ¿qué hubieses hecho para que fueran amigos el resto de niños?", orienta a cada niño en cómo dar solución a un dilema o problema que es importante para el desarrollo del cuento, logra implicar a los niños en la actividad, además de producir respuestas nuevas y propias.

También influye cómo centra la maestra esas respuestas cortas del alumno. Pueden ser ellas quienes completen o den solución a sus preguntas, sin esperar al alumno, pensando por él; o pueden partir de estas respuestas cortas, demandando una argumentación que guíe, matice, o complete, para terminar construyendo conjuntamente una respuesta cercana a la demanda inicial de la maestra que, en principio, se mostraba insuficiente para la maestra. Cuando hacen preguntas y comentarios para ampliar el pensamiento, ayudan a que comprenda la clase de información que su interlocutor necesita y, con el paso del tiempo, empiezan a anticipar el tipo de preguntas que podrían formulársele, y a ofrecer espontáneamente la información requerida.

Centrándonos en el análisis de cada una de las maestras, la maestra A parte de la comprensión de la información del texto, sus enunciados son formulados en condicional para elaborar inferencias, auténticas hipótesis sobre el texto, por ejemplo: "¿si sucede tal cosa, entonces qué creéis vosotros que hubieran hecho o dicho?, ¿quién sería (qué personaje) para darse esta situación?". En cambio el grupo B sólo utiliza el condicional para que cada niño se proyecte sobre el cuento en un ejercicio de aplicación de la imaginación: “¿Qué hubieras hecho tú en esta situación?" (Imagínate que eres el niño del cuento, ¿qué harías?).

El estilo de participación en los grupos A y B tiende a ser asimétrico, pero moderado o negociado, puesto que los niños tienen un amplio margen para enfocar su respuesta, y la posibilidad de valorar las respuestas de otros. Va tomando aspectos del modelo propuesto por Rogoff (1993): animar a los alumnos a escuchar a sus compañeros, proporcionando apoyo para que los niños aprendan cómo hacerlo, y confrontando continuamente determinados enunciados de un interlocutor con el resto del grupo. Los alumnos se refieren tanto a sus compañeros como al profesor, explorando las ideas de sus compañeros y dirigiendo ellos mismos en contados casos la discusión.

Mientras, la maestra $\mathrm{C}$ apenas utiliza el condicional, y muy pocas veces utiliza las inferencias, porque no es su objetivo el repaso y la comprensión del texto como así señala en la entrevista, se conforma con hablar con los niños sobre sugerencias del cuento relacionadas con la vida cotidiana, pero pocas veces lleva estas experiencias, combinándolas (Vygotsky, 1990), hasta desarrollar la imaginación.

La maestra $\mathrm{D}$ desarrolla las inferencias en una tarea que en sus grupos se convierte en rutina, recuperar e interpretar lo que ella percibe como el mensaje central del cuento, el valor esencial por lo que ha sido escrito, sin detenerse en los sucesos importantes que llevan a ese mensaje final o moraleja. 
Aplicando las características de los modelos apuntados por Rogoff y Toma (1997: 25), y diferenciando entre la emergente "Perspectiva de transformación de la participación" y el "Modelo de transmisión de la instrucción", descubrimos que:

- Las maestras A y B intentan seguir la "perspectiva de transformación", consiguiendo puntualmente interacciones de tipo multidireccional, pero con las limitaciones del control cerrado sobre el tópico o tema tratado en cada turno.

- Las maestras C y D se encuentran en la transición de un estilo a otro. Mantienen las interacciones diádicas del modelo tradicional transmisivo para respetar el guión de preguntas que se han propuesto. Al comienzo de cada sesión mantienen un estilo de interacción "asimétrico", ya que asumen el rol de iniciar el intercambio con los mismos tópicos a partir de preguntas abiertas. Y una vez que han intervenido todos sobre los tópicos propuestos, posibilitan, en un segundo momento, el libre desarrollo expresivo de cada niño, incluso el intercambio entre ellos.

La habilidad de la maestra para dar continuidad a las respuestas iniciales del niño se ha mostrado clave, apostando por dar enfoques a la necesidad de contar de los niños, sin salirse de los cauces de la lectura previa. Los resultados apuntan a que utilizar varios tipos de preguntas facilita esta labor (Albanese y Antoniotti, 1997). Las estrategias docentes son gradualmente adoptadas por los alumnos, a través de las habilidades crecientes de la imitación y la escucha.

\section{REFERENCIAS BibLIOGRÁFICAS}

ALBANESE, O. y ANTONIOTTI, C. (1992). The influence of an educator's interactive style on the process of comprenhension in preschool age children. En Wolters y Schnotz (Eds.), Text comprehension and learning 5, 201-209. Amsterdam.

ALBANESE, O. y ANTONIOTTI, C. (1997). Teacher Dialogue Style and Children's Story Comprehension. European Journal of Psychology of Education 12 (3), 249-59.

ANGUERA, T. (1992). Metodología de la observación en ciencias humanas. Madrid. Cátedra.

BAKEMAN, C. y GOTTMAN, R. (1992). Observación de la interacción: introducción al análisis secuencial. Madrid. Cátedra.

BARBIERI, M.S.; DEVESCOVI, A. y BONARDI, P.A. (1987). Línterazione verbale tra bambino ed educatrice durante il racconto di una storia. En S. Mantovani y T. Musatti (Eds.), Adulti e bambini. Educare e Comunicare. Roma. Juvenalia.

BORZONE, A. y ROSEMBERG, C. (1994). El intercambio verbal en el aula: las intervenciones de los niños en relación con el estilo de interacción del maestro. Infancia y aprendizaje, 67-68, 115-132.

BORZONE, A. (2005). La Lectura de Cuentos en el Jardín Infantil: Un Medio Para el Desarrollo de Estrategias Cognitivas y Lingüísticas. Psykhe, 14 (1), 192-209.

BRUNER, J. (1986). El habla del niño: aprendiendo a usar el lenguaje. Barcelona. Paidós. BRUNER, J. (1997). La educación, puerta de la cultura. Madrid. Aprendizaje Visor.

DÍEZ, C.; PARDO, P.; LARA, F.; ANULA, J.J. y GONZÁLEZ, L. (1999). La interacción en el inicio de la lectoescritura. Madrid. CIDE. Ministerio de Educación y Cultura. DÍEZ VEGAS, C. y PARDO DE LEÓN, P. (2000). El trabajo en grupo en el inicio de la escritura. UNED. Madrid. Fundación Universidad-Empresa. 
DÍEZ VEGAS, C. (2002). La interacción social y la construcción del conocimiento en el inicio lectoescritor. Un estudio longitudinal. Tesis doctoral. Madrid. Universidad Nacional de Educación a Distancia.

EDWARDS, D. y MERCER, N. (1988). El conocimiento compartido. Madrid. Paidós y MEC.

FRESQUET, A. (2004). La pregunta como instrumento. Fundamentos de la diversificación curricular. El Boletín Semanal de la Asociación Mundial de Educadores Infantiles, 191.

GONZÁLEZ GARCÍA, J. (2006). Elaboración conjunta de inferencias a partir de cuentos infantiles. Psicología Educativa, 12 (2), 67-89.

GONZÁLEZ GARCÍA, J. (2007). ¿Qué sabemos de las narraciones como construcción social? Investigación en la Escuela, 62 (2), 79-98.

GONZÁlEZ GARCÍA, J. (2008). Debates en el aula de Preescolar a partir de cuentos: Estudio comparado en España y México. Perfiles educativos, 29, 118, 54-78.

GONZÁLEZ GARCÍA, J. (2009). Question like instrument of reading story. International Journal of Hispanic Psychology, 4, 38-53.

GONZÁLEZ GARCÍA, J. (2010). La puesta en discusión de narraciones infantiles. Estudio comparado en España y México. Educación XXI, 35 (1), 141-59.

GONZÁLEZ GARCÍA, J. (2011). Desarrollo de la identidad a partir de experiencias narrativas. Revista Iberoamericana de Educación, 54 (5).

GONZÁLEZ GARCÍA, J. (2012). El maestro que aprende: más allá de teorías implícitas. La coherencia entre objetivos y estilos educativos. Apuntes de Psicología, 30 (1), 5-22.

MERCER, N. (1997). La construcción guiada del conocimiento. Madrid. Paidós.

ORSOLINI, M. y PONTECORVO, C. (1986). Disputare e construire. Processi di conoscenza nell'interazione verbale tra bambini. Età evolutiva, 30, 63-76.

ORSOLINI, M.; PONTECORVO, C. y AMONI, M. (1989). Discutere a scuola: interazione sociale e attività cognitiva. Giornale italiano di Psicología, XVI, 3.

PONTECORVO, C. (1987). Discussing for reasoning: the role of argument in knowledge construction. En E. De Corte, J. Lodewijks, R. Parmentier y P. Span (Eds.). Learning and Instruction. European research in an international context, 1-82. Leuven: Leuven University Press.

PONTECORVO, C. y ORSOLINI, M. (1992). Analizando los discursos de las prácticas alfabetizadoras desde la perspectiva de la actividad. Infancia y Aprendizaje, $58,125-141$.

RODARI, G. (2000). Grámatica de la fantasía. Introducción al arte de inventar cuentos. Buenos Aires. Colihue/Biblioser.

ROGOFF, B. (1993). Aprendices del pensamiento. Barcelona. Paidós.

SÁTIRO, A. y DE PUIG, I. (2000). Jugar a pensar. Recursos para aprender a pensar en educación infantil. Barcelona. Eumo-Octaedro.

SIGNES, C. (2004). Jugando a pensar. Una experiencia en educación infantil. Creamundos. Disponible en: http://personal.telefonica.terra.es/web/crearmundos/. [Consultado: 08-05, 2006].

WELLS, G. (1999). Dialogic Inquiry. London. Cambridge University Press. 